\title{
Téoros
}

Revue de recherche en tourisme

\section{Les intervenants touristiques de l'Estrie, profils comparés et perceptions}

\section{Roger Nadeau}

Volume 16, numéro 1, printemps 1997

La région touristique de l'Estrie / Cantons-de-l'Est

URI : https://id.erudit.org/iderudit/1074922ar

DOI : https://doi.org/10.7202/1074922ar

Aller au sommaire du numéro

Éditeur(s)

Université du Québec à Montréal

ISSN

0712-8657 (imprimé)

1923-2705 (numérique)

Découvrir la revue

Citer cet article

Nadeau, R. (1997). Les intervenants touristiques de l'Estrie, profils comparés et perceptions. Téoros, 16(1), 16-21. https://doi.org/10.7202/1074922ar d'utilisation que vous pouvez consulter en ligne.

https://apropos.erudit.org/fr/usagers/politique-dutilisation/ 


\section{than. \\ LES INTERVENANTS TOURISTIQUES DE L'ESTRIE, PROFILS COMPARÉS ET PERCEPTIONS}

\author{
Roger Nadeau, professeur \\ Géographie et télédétection - Université de Sherbrooke
}

Les donnés livrées ici sont le fruit d'we recherche effectué à l'automne de 1993 avec l'aide de 24 tudiants(es) du Département de gégraphie et telédétection de I'Université de Sherbrooke. Au tolal, 473 entrevues ont été rélisées dans plusieurs regions touristiques du Québec, mais plus porticulierement en Estrie, en Montérégie, en MarricieBois-Francs et en Chaudièr-Appalaches.

\section{SITUATION CONTEXTUELLE ET MÉTHODOLOGIE DE LA RECHERCHE}

L'objectif principal de cette recherche visait à obtenir une meilleure connaissance des intervenants touristiques québécois, de ces hommes et de ces femmes qui font le tourisme au jour le jour, qui sont sur la première ligne de contact avee nos vacanciers et qui ont la passion du travail bien fait. Mais quel est le profil général de tous ces gens? Quelles perceptions ont-ils des structures sensées les encadrer, de l'industrie touristique régionale et nationale, etc... Bien que ces intervenants jouent un rôle tout à fait central et capital au sein de l'industrie, on peut s'étonner de constater qu'il existe fort peu d'études sur le sujet. Même modeste, la contribution de cette étude sera de mettre en lumière certaines réalités souvent méconnues.

Pour y arriver, nous avons consulté des collègues universitaires, des gens du MTO, des Offices municipaux de tourisme et les gens de terrain; ceux-ci nous ayant encouragés à aller de l'avant, nous avons construit l'outil méthodologique principal, le questionnaire, et avons réalisé un prétest. Pour pouvoir répondre à certaines exigences pratiques, le territoire du Québec a été subdivisé en tenant compte de l'origine géographique des étudiants plutồt qu'en reproduisant à l'échelle du Québec la répartition réelle des intervenants touristiques par région (ATR).

Considérant ce résultat étroitement dependant des petits moyens de la recherche, seule l'Estrie peut être analysée comme région; aucune autre région, considérée isolément, n’a assez de cas pour autoriser une étude quantitative. Mais en regroupant les ATR par sous-ensemble, comme ci-haut, certaines comparaisons territoriales deviendront possibles avec l'aide d'outils statistiques appropriées. Pour les besoins du présent article, nous présentons, en parralèle, les résultats pour les intervenants estriens et ceux pout tous les autres secteurs interrogés, sans risquer trop de comparaisons...

Avec le concours de 24 étudiants, nous avons ecrit a 529 intervenants/entreprises touristiques pour leur expliquer la démarche que nous nous préparions à faire et pour les aviser que nous les contacterions 5 ou 6 jours plus tard, pour prendre rendez-vous. L'entrevue avait effectivement lieu sur le site de l'entreprise, la plupart du temps avec le propriétaire, le co-propriétaire ou un cadre supérieur. Pas moins de 500 entrevues ont effectivement eu lieu; 27 questionnaires ont été rejetés. parce qu incomplets, parce que le répondant n'était manifestement pas suffisamment informé ou bien parce que l'entreprise visitée ne s'avérait pas touristique. Cette méthodologie exigeante a permis un taux de réponses de $94,5 \%$ (500/529) ou de $89,4 \%(473 / 529)$ si on ne tient compte que des entrevues valides.

Le rapport de recherche découlant de cette enquête a été publié à la fin de 1996 au Département de géographie et télédétection de l'Université de Sherbrooke. Pour les fins du présent texte, une compilation spéciale a été effectuée afin d'isoler de l'échantillon global les répondants estriens, ce qui nous permettra de comparer les résultats estriens avec ceux de l'ensemble des autres régions.

\section{LE PLAN D'ENTREVUE}

Le questionnaire - et bien șûr l'entrevue se divisent en quatre volets distinets. Les résultats livrés ici le seront donc selon ce même modèle:

\section{VOL.ET I:}

L'intervenant touristique face à la structure nationale qu"est le ministère du Tourisme du Québec (MTO).

\section{VOLET II:}

L'intervenant touristique face à la structure régionale qu'est l'Association touristique régionale (ATR). 


\begin{tabular}{|c|c|c|}
\hline \multicolumn{3}{|c|}{$\begin{array}{l}\text { TABLEAU } 1 \\
\text { Perceptiones des intervenants touristiquess sur quelques variables touchant le MTO. (1993) Profils companés. }\end{array}$} \\
\hline VARIABLES & ESTRIE & GROUPE-TÉMOIN \\
\hline $\begin{array}{l}\text { Relations tréquentes et de qualite avec le MTO } \\
\text { Je connais les services afferts par le MTO } \\
\text { Je ne connais pas les services offerts par le MTO } \\
\text { Je connais peu les services }\end{array}$ & $\begin{array}{l}22,3 \% \\
18,6 \% \\
39,2 \% \\
42,1 \%\end{array}$ & $\begin{array}{l}18 \% \\
26 \% \\
24 \% \\
50 \%\end{array}$ \\
\hline $\begin{array}{l}\text { Lindustrie touristique du } 0 \text { se porte bien } \\
\text { Elle traverse une periode difficile tamporaire } \\
\text { Elle connail des difficultes serieuses } \\
\text { Je ne sais pass }\end{array}$ & $\begin{array}{l}24,4 \% \\
35,4 \% \\
29,22 \% \\
10 \%\end{array}$ & $\begin{array}{l}22 \% \\
39 \% \\
25 \% \\
10 \%\end{array}$ \\
\hline $\begin{array}{l}\text { Je connais bien les programme d'aide du MTO } \\
\text { Je suis assez peu infarme sur ces programmes } \\
\text { Ouels programmes? Connais pas } \\
\text { Le MT0 dispose doune politique claire }\end{array}$ & $\begin{array}{l}11,7 \% \\
57,3 \% \\
30,4 \% \\
0,4 \%\end{array}$ & $\begin{array}{l}14 \% \\
64 \% \\
22 \% \\
0 \%\end{array}$ \\
\hline $\begin{array}{l}\text { La maximisation des retambées, c'est atteint par le MTO } \\
\text { On pourrain faire beaucoup mieux } \\
\text { On fait vraiment piètre figure } \\
\text { Je ne sais pas }\end{array}$ & $\begin{array}{l}3,9 \% \\
56,4 \% \\
15,6 \% \\
24 \%\end{array}$ & $\begin{array}{l}7 \% \\
57 \% \\
15 \% \\
21 \%\end{array}$ \\
\hline $\begin{array}{l}\text { Le MTO fait assez d'effurts pour se faire connaitre } \\
\text { II ne fait pas assez d'efforts pour se faire connaitre }\end{array}$ & $\begin{array}{l}18.3 \% \\
81,6 \%\end{array}$ & $\begin{array}{l}21 \% \\
79 \%\end{array}$ \\
\hline Nombre de repondants (Total: 473 ) & 200 & 265 \\
\hline
\end{tabular}

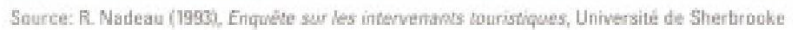

\section{VOLET III:}

L'intervenant touristique face à son entreprise,

\section{VOLET IV:}

Portrait profil de l'intervenant touristique en région.

\section{RÉSULTATS ET ANALYSE}

Le questionnaire ayant servi à l'enquête compte 12 pages et 60 questions. Le rapport de recherche déposé récemment con= tient 90 tableaux et une cinquantaine de figures. On comprendra done volontiers que les rúsultats livrés ici prendront la forme d'un résumé très succinct et que plusieurs variables devront être laissées de côté.

\section{VOLET I:}

\section{L'intervenant touristique face à la structure nationale qu'est le ministère du Tourisme}

Notons au point de départ que sur les 473 répondants consultés, 208 (43.9\%) provenaient de la région touristique de l'Estrie. C'est surtout à partir de cet Échantillon que nous allons travailler dans ce texte. Les répondants non-estriens, au nombre de 265 , constitueront le rgroupe-témoins.
Ce qui ressort le plus nettement du volet I, c'est tres manifestement que les communications et l'information entre le Ministère et les intervenants touristiques sont pour le moins boiteuses et que ce constat se vérifie un peu plus souvent en Estrie qu'ailleurs. Ainsi, bien que les intervenants estriens, dans $22,3 \%$ des cas (comparé à $18 \%$ pour les autres) aient des relations fréquentes et de qualité avec le MTO, il n'en reste pas moins que $39,2 \%$ ne connaissent pas les services offerts par le MTO et que $42,1 \%$ les connaissent "peu*, comparé à 24 et $50 \%$ respectivement pour les autres. Dans le même ordre d'idée, ajoutons qu'en $1993,30,4 \%$ des intervenants touristiques de l'Estrie estimaient ne pas connaître les programmes d'aide du MTO et que $57,3 \%$ se prétendaient wassez peu informes sur ces programmes. Rien d'étonnant donc dans le fait que $81,6 \%$ des personnes consultées en Estrie affirment que le MTO ne fait pas suffisamment d'efforts pour'se faire connaittre. On pourrait citer bien d'autres données qui vont dans le mème sens, mais finalement, l'important est sans doute de faire ressortir un probleme majeur au niveau des communications et au niveau de la diffusion de l'information. Car, enfin, à quoi peuvent bien servir des programmes ou des supports du MTO si les principaux concernés, les intervenants touristiques, n'en sont pas informés? Avec l'avènement des ATR, on avalit compris que cette fonction informa- tion serait dorénawant assuméc par cette nouvelle structure. Si on en croit les don= nées citées précédemment, on doit en déduire que ce relais se fait plutôt laborieusement..

Notons quelques autres point d'intérêt:

- En 1993, les gestionnaires consultés identifiaient comme suit les principales faiblesses du Québec au plan touristique: a) Lacunes en publicité promotion - marketing; b) Les nombreuses taxes; c) Les coûts - prix du produit; d) Problème de concertation entre les personnes concernés - sans oublier l'état du réseau routier, le climat incertain, la signalisation, le manque d'information,... etc...

- $60,3 \%$ des Estriens interrogés estiment que le MTO s"acquitte plutot bien de sa tâche de maximiser les retombées économiques du tourisme, comparé à $64 \%$ pour le groupe-témoin.

- $63,0 \%$ des intervenants qui ont des relations fréquentes et de qualité avec le MTO connaissent les services qu'il offre. Ce pourcentage tombe à $14,7 \%$ chez ceux qui n'ont pas ou peu de contacts avec le MTO.

Par le biais d'une question ouverte, on demandait aux intervenants d'identifier les services ou le programmes qui n'existent pas mais que le MTO devrait offrir. La réponse qui revient le plus souvent, surtout en Estrie et en Montérégie, donne à réfléchir: leur premier souhait, c'est d'être mieux informé - ou tout simplement, d'être informé tout court. Que ce soit là la principale revendication des intervenants touristiques à l'endroit du MTO, a de quoi surprendre.

\section{VOLET II:}

\section{L'intervenant touristique et la structure régionale, I'ATR}

Le volet II s'intếresse d'une façon particulière aux Associations touristiques rếgionales (ATR) et plus spécifiquement à I'Association touristique de l'Estrie (ATE). A noter ici que seuls les membres en règle d'une ATR étaient interrogés sur ce volet II, cela afin de ne conserver que les intervenants directement concernés. Sur l'échantillon total (473 
cas), $68,02 \%$ des répondants étaient membres d'une ATR. En Estrie, 61,0\% de nos répondants étaient membres de l'ATE. En nombre absolu donc, l'échantillon total compte 311 membres d'ATR, dont 121 en Estric.

Le tableau 2 fait ressortir quelques points de comparaison entre les membres de Tourisme Estrie et les autres membres d'ATR relatif's à leur comportement, perception ou évaluation associées au fonctionnement de cette structure régionale. On notera, dans un premier temps, que les membres d'ATR ont une bien meilleure perception de cette structure qu'ils n'en avaient pour le MTO, sans doute parce qu'elle est plus proche d'eux. On peut cependant observer que les lacunes liées à la diffusion de l'information sont, ici aussi, bien présentes et ce, malgré le fait que la grande majorité des membres reçoivent de leur ATR un «bulletin d'informations: à des intervalles diverses ( $75 \%$ de l'ensemble). On note donc qu'en 1993, 31,6\% des membres de Tourisme Estrie se disaient atrès bien informés" des services que pouvait offrir l'organisme, comparé à $46,3 \%$ pour les membres provenant du groupe-témoin. $\AA$ l'autre extrémité, $23,7 \%$ des membres estriens se disaient apas du tout informés $\%$, comparativement à $11,7 \%$ ailleurs. Sur l'ensemble de l'échantillon done, cette lacune, (en 1993) semble plus sévèrement ressentie en Estrie qu'ailleurs. Il en est de même pour le faible taux de participation des membres à leur Assemblée générale annuelle. Si la composante de base de la vie démocratique d'un organisme repose sur l'implication de ses membres lors des AGA, on peut sans doute s'interroger sérieusement quand moins de $10 \%$ des membres participent régulièrement à l'Assemblée générale.

Il n'en demeure pas moins que selon nous, comme l'exprimait un ancien ministre du tourisme du Québec, monsieur Marcel Léger.... «Dans le domaine du tourisme, les ATR sont la plus belle invention depuis la roues. La structure, en effet, permet beaucoup de souplesse et d'efficacité: c'est souvent le facteur humain qui explique les variations de performance d'une ATR à l'autre mais il ne faut non plus laisser de côté l'implication concrête et effective de l'ensemble des membres dans la vie et le fonctionnement de leur organisme, car en fait, une ATR, c'est un outil que les intervenants

\begin{tabular}{|c|c|c|}
\hline \multicolumn{3}{|c|}{$\begin{array}{l}\text { TABLEAU } 2 \\
\text { Perceptiens des intervenamts touristiques menabres de leur ATR sur quelques variables touchant la vie de l'ATR. (1958) Profils comparess }\end{array}$} \\
\hline VARIABLES & ESTRIE: & GROUPE-TËMOIN \\
\hline Selon mai, l'ATR est l'interloc uteur privilégié du MTO & $63,3 \%$ & $65,4 \%$ \\
\hline $\begin{array}{l}\text { Naus sommes très bè̈en informess de services alferts par l'ATR } \\
\text { Naus ne sommes pas du tout informés de ces services }\end{array}$ & $\begin{array}{l}31,6 \% \\
23,7 \%\end{array}$ & $\begin{array}{l}46,3 \% \\
11,7 \%\end{array}$ \\
\hline Nous recenons un Bulletin d'informations de l'ATR' & $71,4 \%$ & $78,8 \%$ \\
\hline $\begin{array}{l}\text { Je suis très satisfait de l'information sur los programmes } \\
\text { Je suia plutot satisfait } \\
\text { Je suis plutót insatisfait } \\
\text { Je suis tres insatisfait. }\end{array}$ & $\begin{array}{l}6,06 \% \\
50,0 \% \\
30,3 \% \\
13,6 \%\end{array}$ & $\begin{array}{l}9,0 \% \\
50,9 \% \\
30,6 \% \\
9,5 \%\end{array}$ \\
\hline $\begin{array}{l}\text { Je ne manque jamais une AGA de mont ATR } \\
\text { Je na ai jamais participé à une AGA de mon ATR }\end{array}$ & $\begin{array}{l}9,7 \% \\
55,6 \%\end{array}$ & $\begin{array}{l}18,2 \% \\
46,0 \%\end{array}$ \\
\hline $\begin{array}{l}\text { Pour le adéveloppement du praduita, mon ATR est très dynamique } \\
\text { Elle est plutōt absente du volet dévelappement } \\
\text { Elle fait preuve d'un leadership occasionnal } \\
\text { Mon ATR ne s'intèresse qu'áa la publirité }\end{array}$ & $\begin{array}{l}14,7 \% \\
23,7 \% \\
477 \% \\
14,7 \%\end{array}$ & $\begin{array}{l}19,4 \% \\
26,1 \% \\
41,7 \% \\
12,8 \%\end{array}$ \\
\hline $\begin{array}{l}\text { Grâce à I'ATR, les intervenants travallent plus ensembla } \\
\text { Avec raTR, il y a eu tarn peu de progres a la concertation }\end{array}$ & $\begin{array}{l}23,9 \% \\
32,6 \%\end{array}$ & $\begin{array}{l}23,8 \% \\
30,2 \%\end{array}$ \\
\hline 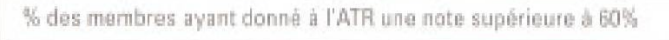 & $67,9 \%$ & $61,7 \%$ \\
\hline $\begin{array}{l}\text { Selon mai, lindustrie touristigue en région va trés bien } \\
\text { Selon mai, ça va bien } \\
\text { Selon mai, c'est asser difftcile } \\
\text { Selon mai, ça va carrément mal }\end{array}$ & $\begin{array}{l}5,5 \% \\
47,9 \% \\
4,1 \%\end{array}$ & $\begin{array}{l}5,2 \% \\
47,8 \% \\
4,4 \%\end{array}$ \\
\hline Non, je ne connais pas l'ATRAa & $81,6 \%$ & $79,6 \%$ \\
\hline Nombre de répondants (Totak 311 membres) & 121 & 150 \\
\hline
\end{tabular}

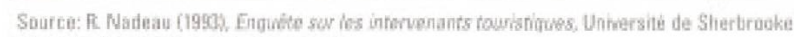
(1) $A G A$. Assemmilè qénerale annuells

touristiques se donnent afin de développer des services de meilleure qualité et pour défendre leurs intérêts professionnels.

Rappelons quelques informations supplémentaires découlant de la recherche effectuée auprès de ces intervenants:

- Être membre d'une ATR, c'est d'abord un geste de solidarité avec l'Industrie $(49,3 \%$ de l'échantillon total). C'est aussi s'assurer plein de services $(36,9 \%)$ et voir ses intérêts bien défendus $(28,7 \%)$. Par contre, $28,7 \%$ ne seraient pas membres s'ils n'y étaient pas obligés tandis que $13,1 \%$ prétendent être membres spour ne pas être boycottés\%. (Les pourcentages dépassent $100 \%$ car plusieur's réponses étaient possibles).

- 57,2\% des membres de Tourisme Estrie estimaient en 1993 que leur ATR était un actif favorable en faveur de la concertation contre $42,7 \%$ qui pensaient le contraire. Les membres de l'extérieur de l'Estrie donnaient un portrait à peu près identique.
- Quand on demande aux membres quelles suggestions ils font aux ATR, quelles améliorations ils souhaiteraient, les cinq réponses qui reviennent le plus souvent sont les suivantes:

- Mettre plus d'efforts et de régularité à la transmission de l'information.

- Favoriser une plus grande concertation entre les intervenants, entre les régions et les sous-régions et entre tous les organismes s'occupant de tourisme.

- Que les efforts et la présence de I'ATR soient manifestes sur l'ensemble de la région touristique et que l'on travaille autant avec les PME qu"avec les millionnaires.

- Que l'on reconnaisse la place et le rôle des Offices municipaux de tourisme.

- On note aussi une préoccupation au niveau de la formation, d'un effort plus important au niveau de la publicité en général, et d'une signalisation routière et touristique moins onéreuse et de qualité. 
TABLEAU 3

Perceptions des interventents touristiques sur qualques variables touchant la vie de leur entreprise. (1993) Prolils comparés.

\begin{tabular}{|c|c|c|}
\hline VARIABLES & ESTRIE & GROUPE-TEMOIN \\
\hline 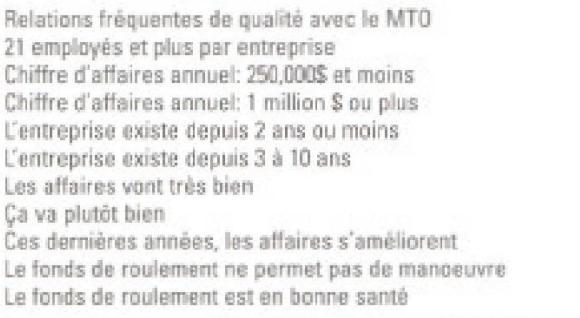 & $\begin{array}{l}22,3 \% \\
20,8 \% \\
54,2 \% \\
21,2 \% \\
15,6 \% \\
31,9 \% \\
29,8 \% \\
57,2 \% \\
56,6 \% \\
31,4 \% \\
18,3 \%\end{array}$ & $\begin{array}{l}18 \% \\
26,0 \% \\
48,0 \% \\
22,9 \% \\
7,8 \% \\
39,8 \% \\
27,6 \% \\
55,4 \% \\
47,4 \% \\
30,8 \% \\
16,0 \%\end{array}$ \\
\hline $\begin{array}{l}\text { Nous sommes tres bien signalises fbleu) } \\
\text { Natre entreprise est signalise de façon artisanale } \\
\text { Aucune signalisation }\end{array}$ & $\begin{array}{l}26,6 \% \\
48,7 \% \\
24,1 \%\end{array}$ & $\begin{array}{l}40.2 \% \\
37,7 \% \\
22,1 \%\end{array}$ \\
\hline $\begin{array}{l}\text { Notre personnel est trés professionnell } \\
\text { Persannel qualifie mais ill y a parfois des falles } \\
\text { Notre personnel aurait besoin de formation }\end{array}$ & $\begin{array}{l}45,0 \% \\
47,6 \% \\
7,2 \%\end{array}$ & $\begin{array}{l}50,15 \\
44,75 \\
5,3 \%\end{array}$ \\
\hline $\begin{array}{l}\text { Nous ne nous sommes jamais inderessis aux Grands Prix } \\
\text { Nous avons déja soumis notre candidature }\end{array}$ & $\begin{array}{l}60,4 \% \\
12,5 \%\end{array}$ & $\begin{array}{l}52,2 \% \\
19,1 \%\end{array}$ \\
\hline $\begin{array}{l}\text { Natre clientẻle est } 100 \% \text { touristique } \\
\text { Nous faisans un efffart régulier en publicité } \\
\text { Nas emplayes sont syodiqués } \\
\text { Nous avons obtenu une aide gouvemementale recemment }\end{array}$ & $\begin{array}{l}23,3 \% \\
43,3 \% \\
7,4 \% \\
34,8 \%\end{array}$ & $\begin{array}{l}30,1 \% \\
47,2 \% \\
8,7 \% \\
40,3 \%\end{array}$ \\
\hline Nombre de répondants (Total: 473) & 208 & 265 \\
\hline
\end{tabular}

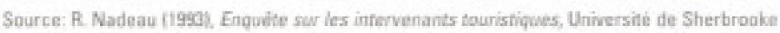

- Dans l'ensemble, les membres de l"ATR de l'Estrie donnent une meilleure note à leur ATR que ne le font les autres.

- Il existe une relation très étroite, dans l'ensemble, entre la perception des membres au sujet de la concertation et l'évaluation qu'ils font de leur ATR. Ainsi, chez les gens qui estiment que la concertation a connu un net progrès chez eux avec $\mathrm{I}^{+}$ATR, $89,4 \%$ cotent cette dernic̀re à $60 \%$ ou plus. Par contre, ce pourcentage descend à $22,3 \%$ chez ceux qui estiment que cette concertation n'a pas progressé, ou même qu'elle a régressé.

- On entend souvent des commentaires faits par de petits entrepreneurs touristiques qui estiment que leur ATR n'en at que pour les entreprises millionnaires et prestigieuses et que les plus petites sont livrées à elles-mêmes. On pourrait done penser que les grandes entreprises donneraient des meilleures notes it leur ATR que les plus petites. Or, si on en croit notre échantillon, ce n'est pas le cas. Les patrons des entreprises ayant un chiffre d'affaires de $1000000 \$$ ou plus annuellement donnent à leur ATR une note de $60 \%$ ou plus dans $47,6 \%$ des cas seulement, comparé a $54,8 \%$ dans le cas des entreprises plus petites.
- $84,4 \%$ de nos répondants disent que leur entreprise à eux va «bien ou wres bien $\%$, mais ils estiment que dans leur région, il n'y a que $53,5 \%$ des entreprises qui peuvent en dire autant et si on va à l'échelle nationale, il n'en reste plus que $25,8 \%$ à penser que notre industrie se porte abiens ou utrès biens. On prenait presque pour acquis que les gens avaient l'habitude de penser que l'herbe était toujours plus verte chez le voisin. On a ici l'exemple du contraire! Voila un beau sujet de thèse pour les sociologues sintéressant aux études de perception.

\section{VOLET III:}

\section{L'intervenant face à son entreprise}

L'étude du volet II ne tenait compte que des répondants membres d'une ATR. Avec le volet III, nous revenons à l'échantillon total, soit aux 473 cas interviewés. Cette partie de l'étude porte spécifiquement sur l'entreprise touristique et sur quelques facettes de son environnement.

Signalons d'abord qué nos répondants sont bien places pour nous parler de leur entreprise: $49,1 \%$ sont les propriétaires/co-propriétaires et $41,7 \%$ sont les cadres supérieurs. Il s'agit d'autres employés dans seulement $9,1 \%$ des cas.
Notons aussi que nous avons affaire à des entreprises privées dans $69,1 \%$ des cas.

Le tableau 3 fait surtout ressortir que les entreprises des régions gravitent dans un environnement assez semblable et que, à part quelques rares exceptions, les ecarts entre l'Estrie et le groupe-témoin semblent plutôt faibles. En fait, l'ccart le plus marqué touche à la signalisation: lat signalisation ableue (touristique) du ministère des Transports était beatucoup moins généralisée en Estric en 1993 qu'elle ne l'était ailleurs $(26.6 \%$ versus $40,2 \%)$. Peut-être parce que les entreprises estriennes sont, dans l'ensemble, de plus petite taille, ont un chiffre d'affaires légèrement inférieur et ont, en moyenne. un peu moins d'ancienneté. Par ailleurs. en 1993, c'est en Estrie que les entrepreneurs sentaient le plus les affaires $s^{4}$ anéliorer $(56,6 \%$ versus $47,4 \%)$.

On note aussi dans ce tableau que dans l'ensemble, les entreprises vont bien ou plutôt bien, et que cette performance va en s'ameliorant. Elles sont cependant fragiles car dans bien des cas, le fond de roulement ne laisse $\mathrm{d}$ peu près aucune marge de manoeuvre à l'entrepreneur. Selon notre echantillon, seulement $17 \%$ des entreprises seraient en très bonne santé à cet égard.

- En ce qui a trait au nombre d'emplois crés par entreprise, la mediane se situe à 7. Quant au chiffre d'affaires, la médiane se situe à 250000 s.

- $16,6 \%$ des entreprises touristiques estriennes sont sans but lucratif (OSBL) compare i $21,4 \%$ ailleurs.

- Selon nos répondants, pour améliorer le rendement des entreprises, les remèdes privilégiés seraient les suivants:

- Plus de publicité

- Une signalisation adéquate

- Un meilleur partenariat et une concertation reelle

- La permanence des employés

- Un meilleur amenagement du site

- Abolition des taxes

- Formation du personnel

- $36,5 \%$ des entreprises comptant 20 employés ou moins ont obtenu une 


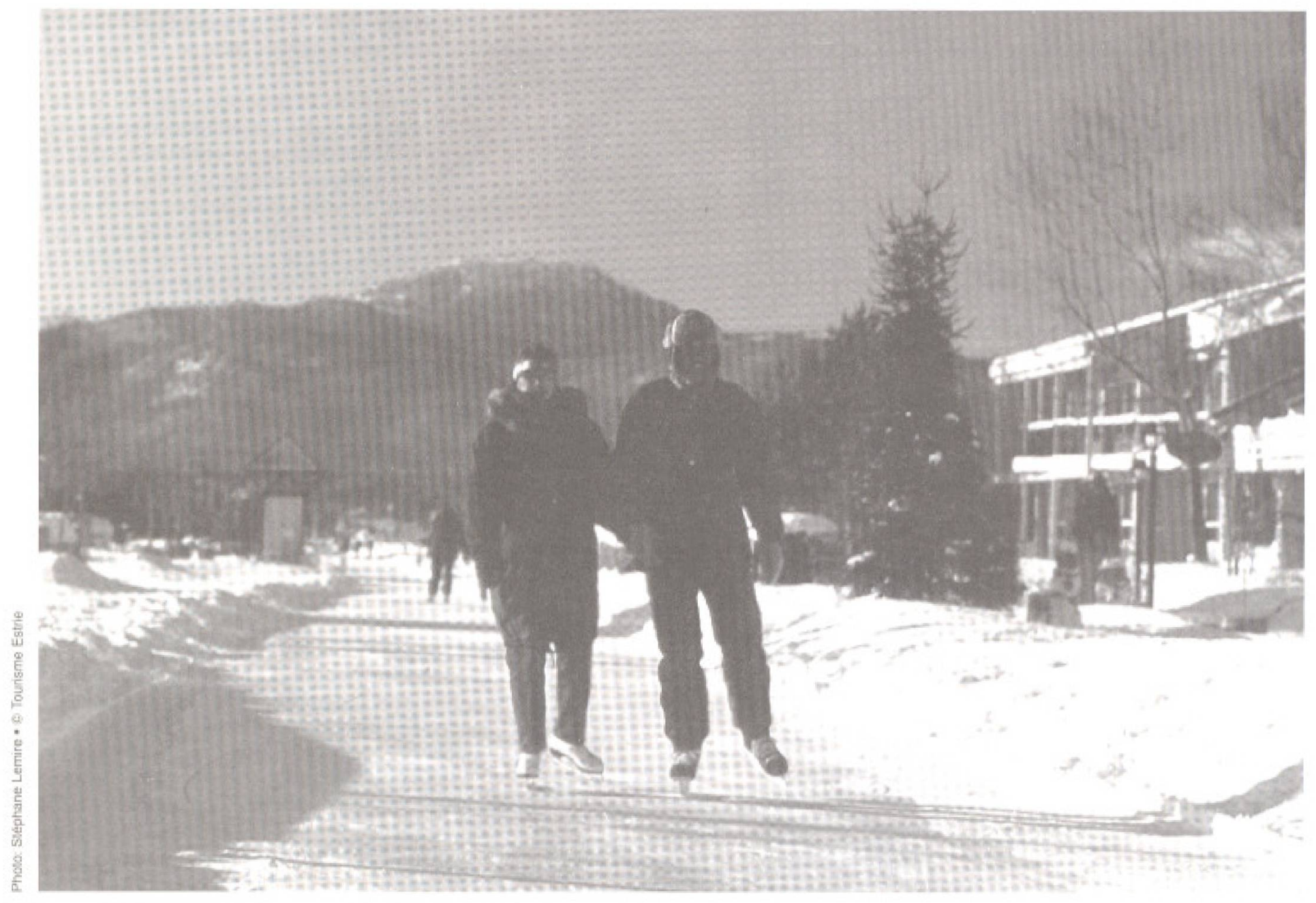

aide gouvernementale quelconque au cours des cinq demières années (vu de 1993). Les entreprises complant 41 employés ou plus avaient reçu un support gouvememental dans $51,5 \%$ des cas.

- $32 \%$ des principaux patrons des entreprises ont 35 ans ou moins (en 1993) et $7.5 \%$ seulement auraient 55 ans ou plus.

- a priori, nous aurions cru que les cadres des plus grosses entreprises seraient notablement plus âgés. Pạs du tout! Bien au contraire; $90 \%$ des cadres répondants associés à des entreprises comptant 100 employés ou plus ont 45 ans ou moins.

- $75,1 \%$ de nos répondants ont fait des études collégiales ou universitaires. Dans les groupes d'entreprises comptant plus de 20 employés. ce pourcentage atteint $86,5 \%$.
- Il y a une relation tres nette entre la taille de l'entreprise et le fait de prendre ou pas des vacances. Ainsi, dans les aboitess ne comptant qu'un employé ou deux, on ne prend des vacances qu'une année sur deux $(49,4 \%)$. Par contre, dans les entreprises qui comptent plus de $20 \mathrm{em}-$ ployés, les principaux gestionnaires. prennent des vacances dans une proportion de $85 \%$.

- Parmi les entreprises qui font un effort soutenu au plan publicitaire. $36,5 \%$ ont présenté dans le passé leur candidature aux Grands Prix du tourisme régional. Ce pourcentage tombe à $14,3 \%$ chez celles qui ne font qu'une publicité occasionnelle et à $8,8 \%$ chez celles qui n'en font pas, ou rarement.

- En haute saison, la valeur médiane en ce qui concerne le nombre d'heures travaillées hebdomadairement par nos répondants se situe à 55 heures. En basse saison. la valeur médiane s'arrête tout de mêne à 40.7 heures, ce qui constitue tout de même des semaines fort bien remplies.

\section{VOLET IV:}

Profil socio-économique de l'intervenant touristique en région

Nous terminons en traçant un bref profil socio-économique de nos répondantsintervenants touristiques, que nous devrions connaître un peu mieux présentement

- 45,3\% des personnes interviewées étaient de sexe féminin et $54,6 \%$ de sexe masculin.

- $31.8 \%$ de nos répondants estriens avaient 35 ans ou moins en 1993 ( $30.8 \%$ chez le groupe-témoin): par ailleurs, $26,9 \%$ avaient 46 ans ou plus $(31.7 \%)$ 
- Parmi les répondants estriens, 38,3\% avaient moins de 5 ans d'ancienneté au sein de l'industrie touristique, comparé à $34,7 \%$ pour ceux provenant des autres régions.

- $53.9 \%$ des intervenants touristiques de l'Estrie travaillaient 55 heures/semaine en haute saison. Il n'y en avait en fait que $26,9 \%$ qui travaillaient 45 heures/semaine ou moins. En basse saison, $44,0 \%$ de nos répondants estriens étaient au boulot pendant plus de 45 heures/semaine.

D'après notre échantillon donc, les entrepreneurs oeuvrant en Estrie en 1993 Étaient un peu plus jeunes que leurs collègues des régions avoisinantes: ils comptaient aussi moins d'années d'ancienneté dans le domaine touristique et leur semaine de travail était nettement plus longue, spécialement en xbasse saison.

- Dans l'ensemble, les intervenants touristiques sont très scolarisés: les trois quart ont fait au moins des études collegiales. Par contre, ces études, dans la très large majorité des cas n'étaient pas orientées vers le tourisme car, dans les faits, un peu moins de $20 \%$ de ces répondants avaient fait des études dans le domaine du tourisme avant d'y venir travailler. La majorité des gestionnaires rencontrés $(53,0 \%$ en Estrie et $49,2 \%$ ailleurs) ont appris le métier ssur le terrain\%. Soulignons aussi que moins de $1 \%$ de nos répondants signalent avoir des problèmes de formation. Dans ces rares cas, les besoins sont dans les domaines suivants: gestion, anglais, accueil, marketing, informatique, relations publiques, animation, comptabilité...

- $32,7 \%$ des chefs d'entreprises consultés n'ont pas pris de vacances l'année précédente à l'enquête. Dans $49,0 \%$ des cas, la raison invoquée est ale manque de temps\%, ou encore, ale manque de temps et d'argents, dans $23,2 \%$ des cas... et le amanque d'argents pour $11,6 \%$ d'entre eux.

- Pour ceux qui prennent des vacances, $22,4 \%$ demeurent dans leur région d'origine, Les autres passent essentiellement leurs vacances au Québec et aux États-Unis. Le Canada, l'Europe et les Antilles ne ramassent ensemble que $18,8 \%$ des adhésions.
- Sur l'échantillon total, $68,3 \%$ des répondants estiment qu'ils sont bel et bien des intervenants touristiques, alors que $19,5 \%$ ne le seraient que xjuste un peum. Il en reste donc $11,7 \%$ qui ne croient pas mériter ce titre.

\section{CONCLUSION}

Voilà pour l'essentiel des données recueillies qui peuvent prendre place dans un texte aussi bref. Les personnes désirant fouiller davantage cette question pourront peut-être prendre connaissance du rapport initial de recherche. Pour notre part, nous croyons que cette première recherche générale sur les intervenants touristiques du Québec, malgré ses imperfections, permet de mieux appréhender certains volets de la réalité touristique du Québec des régions et de l'Estrie plus particulièrement. Nous sommes bien sûr loin d"avoir fait le tour de toutes les questions utiles mais déjà certaines pistes s'imposent. Par exemple, les lacunes majeures observées au niveau de la transmission de l'information à ces gens de terrain que sont les intervenants touristiques devraient susciter des questionnements responsables et engageants. Il en est de même pour le taux anémique de participation aux Assemblées générales annuelles des ATR, l'instance par excellence de la vie démocratique de ces organismes. Beaucoup de questions donc mériteraient d'être approfondies davantage et cette recherche se voudrait surtout un point de départ à une meilleure compréhension du vécu sur le terrain.

Bien évidemment, cet ébauche de portrait vaut pour l'automne 1993. Le sondage transmet un instantané d'une situation qui prévaut à un moment donné dans le temps et dans l'espace. Mais le monde du tourisme se caractérise par sa créativité et son dynamisme. Qu'en est-il donc des intervenants touristiques quatre ans plus tard? Bien sûr, l'industrie a grandi; elle a mûri, elle se professionnalise. Mais, tout n'a pas changé: en mai 1996, l'ATR de l'Estrie tenait son Assemblée générale au Mont Mégantic et on n'y comptait toujours que $9,03 \%$ des membres $(42 / 465) \ldots$

\section{BIBLIOGRAPHIE}

NADEAU, Roger (1996), Les iniervenanis touristiques du Québec. Profils es perceptions des chef d'enreprizes en region. Departement de gégraphie et télédétection. Université de Sherbrooke, Sherbrooke, 286 pages

\section{REMERCIEMENTS}

Chantal DUFORD et

Daniel BOSSÉ (traitement informatique) 